\title{
Redefinición territorial pos COVID-19: resiliencia frente a riesgos y desequilibrios en los modelos urbano-rurales
}

\author{
Sergio Salazar-Galán. Universidad de Sevilla, Sevilla, España. \\ Emilio Mascort-Albea. Universidad de Sevilla, Sevilla, España. \\ Domingo Sánchez-Fuentes. Universidad de Sevilla, Sevilla, España.
}

RESUMEN | La presente Tribuna reflexiona sobre los efectos de la alteración de los ecosistemas y la biodiversidad en el incremento de problemáticas sanitarias y ambientales relacionadas, según evidencia la crisis mundial por la COvid-I9. La generalización de modelos urbanos densamente poblados y en creciente expansión ha propiciado la contaminación ambiental, así como el incremento del riesgo frente a enfermedades e inundaciones. Igualmente, la industrialización de la cadena alimenticia ha favorecido la aparición de enfermedades como diabetes, así como de patógenos que provocan enfermedades infecciosas. Con la globalización, estas últimas se han transmitido rápidamente desde zonas rurales a poblaciones urbanas, en una acelerada expansión. Consecuentemente, se llama a una redefinición de las relaciones campo-ciudad en clave biorregional, y desde una aproximación socio-ecológica, para mantener y restaurar la estructura ecológica de soporte. Con ello se podría fortalecer la resiliencia territorial, a través de una configuración modular, flexible y policéntrica.

PALABRAS CLAVE | relación campo-ciudad, regionalización, transformaciones socioterritoriales.

ABSTRACT | The present Tribune reflects on the effects of the alteration of ecosystems and biodiversity on the increase of related health and environmental problems, as evidenced by the global crisis caused by COVID-19. The generalization of densely populated and increasingly expanding urban models has led to environmental pollution and an increased risk of disease and flooding. Similarly, the industrialization of the food chain has favored the appearance of diseases such as diabetes and pathogens that cause infectious diseases. With globalization, the latter has been rapidly transmitted from rural areas to urban populations, allowing rapid expansion. Consequently, there is a call for a redefinition of the countryside-city relationship from a bioregional perspective and from a socio-ecological approach to maintain and restore the ecological support structure. This could strengthen territorial resilience through a modular, flexible, and polycentric configuration.

KEYWORDS | countryside-city relationship, regionalization, socio-territorial transformations. 


\section{La COVID-19 y la crisis ambiental}

Es un hecho que los ecosistemas constituyen el soporte fundamental para la vida humana y todas las formas de vida del planeta Tierra. A pesar de ello, la Evaluación de los Ecosistemas del Milenio (Millennium Ecosystem Assessment, 2005) concluyó que desde la segunda mitad del siglo xx los seres humanos hemos provocado transformaciones aceleradas y perjudiciales para nuestro hábitat. En el Antropoceno, esos cambios sustanciales y crecientes sobre los ecosistemas han causado una gran pérdida de biodiversidad y el cambio climático (Vitousek et al., 1997). La combinación del crecimiento poblacional junto con la pérdida de ecosistemas y biodiversidad ha facilitado la transferencia de patógenos de animales a personas con un promedio de una nueva enfermedad infecciosa en los humanos cada cuatro meses. Esta situación se ha exacerbado debido a los cambios en la temperatura, la humedad y la estacionalidad, afectando la supervivencia de los microbios en el ambiente (United Nations Environmental Programme [UNEP], 2016). Es esperable entonces que las epidemias, e incluso las pandemias, sean más frecuentes debido al cambio climático.

Los cambios planetarios, que amenazan nuestra propia existencia como especie, son el resultado de un modo de producción que fue concebido sin límites físicos para su expansión y reproducción. Por ello, desde los principales organismos internacionales se han impulsado agendas como la del "desarrollo sostenible", en tanto eje vertebrador de factores sociales, económicos y ecológicos para la toma de decisiones en el presente, con visión de futuro. No obstante, dicho paradigma ha mostrado ser insuficiente para evitar lo que se ha denominado "crisis ambiental" (Kapecki, 2020), ya que sus principios fundamentales se basan en la continuidad de los actuales medios de producción y consumo para mantener un crecimiento económico progresivo (Declaración Vasca, 2016; Spaiser et al., 2017). En ese contexto, ha aparecido la pandemia producida por la covid-19, la cual ha generado una crisis humanitaria en gran parte de los países del mundo, que a su vez está teniendo impactos socioeconómicos severos al provocar una disrupción en las inercias productivas y de los hábitos culturales y relacionales desde principios de 2020. Los efectos de dicha enfermedad han puesto de manifiesto el nivel de vulnerabilidad social existente debido a que están convergiendo, de manera sinérgica y acumulativa, los efectos de decisiones políticas de orden planetario - tales como el debilitamiento de la inversión pública para la prestación de servicios sociales esenciales, la priorización de la economía de mercado-, el uso de combustibles fósiles y los impactos sanitarios y ambientales provocados por los modelos de desarrollo urbano y rural preponderantes.

A la COVID-19 se le está llamando "sindemia", por su sinergia con otras epidemias y sus consecuencias por coocurrencia y la interacción biológica y social entre ellas (Gravlee, 2020). La presente Tribuna tiene por objeto reflexionar sobre esta última cuestión, en particular sobre las interrelaciones existentes entre riesgos biológicos y ambientales asociadas a los actuales modelos de desarrollo urbano y rural, y propone avanzar en estrategias potenciales en el mantenimiento y restauración de la estructura ecológica de soporte y en el incremento de la resiliencia socioecológica. 


\section{Modelo urbano}

Los valores fundamentales que soportan el paradigma urbanístico moderno son tanto el valor social, que nace como respuesta a los graves problemas de salubridad que impulsó la Revolución Industrial, como el crecimiento poblacional y económico. De acuerdo con una publicación de Naciones Unidas (United Nations, Department of Economic and Social Affairs [UN-DESA], 2019), el 55\% de la población mundial vive en zonas urbanas y se espera que dicha proporción aumente a un $68 \%$ para 2050. El rápido desarrollo urbano ha generado la alteración del paisaje natural y la pérdida de ecosistemas. Entre los principales factores causantes, se puede destacar la impermeabilización del suelo, la construcción masiva de infraestructuras y la expansión de las tierras de cultivo. Todo esto se ha producido en muchos casos de manera no planificada y ha provocado algunas de las siguientes amenazas y riesgos asociados a la salud humana: infravivienda, hacinamiento, contaminación del aire y del agua, servicios sanitarios y de recogidas de residuos sólidos inadecuados (Moore et al., 2003). Los citados autores también han reportado que la expansión urbana hacia hábitats silvestres ha dado lugar a un aumento significativo del riesgo biológico con enfermedades como la leishmaniasis, la leptospirosis y la fiebre amarilla. La contaminación atmosférica urbana es la causante de una tasa de mortalidad entre 6 a 9 millones de personas/año y una pérdida de esperanza de vida de tres ańos en promedio (Lelieveld et al., 2020). Igualmente, la expansión acelerada de los asentamientos humanos ha originado riesgos asociados a fenómenos naturales como las inundaciones, que para el período 2000-2019 se han duplicado, sumando el $44 \%$ de todos los desastres reportados a nivel mundial, con una afectación de 1.6 billones de personas (Centre for Research on the Epidemiology of Disasters \& United Nations Office for Disaster Risk Reduction [CRED \& UNDRR], 2020).

El actual potencial de transmisión y expansión de enfermedades infecciosas como la covid-19 en los ambientes urbanos masificados se debe a causas como la alta conectividad social, los patrones de movilidad y las rutinas diarias laborales y sociales que favorecen el contagio por vía aérea, o a través del contacto directo o indirecto. De acuerdo con Megahed y Ghoneim (2020), más allá del desarrollo de tratamientos médicos específicos para enfrentar dicha enfermedad, se conseguirán soluciones integrales y de carácter transversal a través de la implementación de nuevos paradigmas en el diseño del entorno físico y construido urbano. De este modo, la arquitectura y el urbanismo vuelven a recibir por parte de la sociedad un papel fundamental en la generación de espacios colectivos saludables, que reduzcan el impacto de enfermedades contagiosas, y a su vez territorios resilientes que integren todos los riesgos ambientales.

Resulta fundamental entonces ir a la raíz de las actuales problemáticas de manera integral, y reconectar los espacios urbanos y rurales vinculados, impulsando la gestión en torno a biorregiones como eje estructurante para mantener el equilibrio socioecológico territorial (Prats et al., 2017). 


\section{Modelo rural}

En el Antropoceno, el desarrollo rural ha estado principalmente enfocado a la producción de alimentos y materias primas, y su deslocalización hacia la globalización ha generado una ruptura en las relaciones campo-ciudad. El rápido crecimiento de la población a nivel global ha tenido efectos en una mayor demanda de alimentos, y con ello se ha dado la transformación de un $40 \%$ de la superficie terrestre para la agricultura y la ganadería (Foley et al., 2005). La producción agroindustrial es el principal motor de cambios del paisaje natural y de erosión del suelo, siendo el sector económico que mayor consumo de agua realiza, supone la principal fuente de contaminación hídrica y de emisiones de gases de efecto invernadero (GEI) y constituye una de principales causas de pérdida de biodiversidad (Bruinsma, 2003). La agricultura es el primer sector afectado por las sequías en todo el mundo y, a esa escala, los estudios han concluido sistemáticamente que frente al cambio climático cabe esperar efectos negativos importantes (Food and Agricultural Organization [FAO], 2015).

En contraste con lo anterior, la industrialización de la cadena alimenticia ha propiciado una incidencia global en el sobrepeso y obesidad, y en relación con ello, en muchos países en desarrollo ha incrementado la prevalencia de la diabetes y sus complicaciones en la salud humana (Yach et al., 2006). Igualmente, en la actualidad se desperdicia un tercio de la producción global de alimentos y miles de personas mueren al año por hambre (Elkins et al., 2019).

Por todo ello, es necesario analizar la influencia que sobre la salud humana y la de los ecosistemas está causando el actual modelo agroindustrial. Wallace et al. (2020) resaltan que el acaparamiento de tierras en los últimos bosques primarios y parcelas de cultivo de pequeños propietarios, la deforestación, o el comercio de especies exóticas, están favoreciendo el contacto humano con nuevos patógenos, que antes se mantenían confinados por las ecologías forestales de larga evolución. Asimismo, dichos autores señalan que su transmisión se ve agravada por el desarrollo genético de monocultivos de animales y plantas, al eliminarse cualquier cortafuegos inmunológico y al proporcionar un suministro continuamente renovado para la evolución de la virulencia. El Ébola, el Zika y la Fiebre Amarilla han reaparecido recientemente debido a la tala de árboles, la minería y la agricultura intensiva en los bosques neotropicales, al igual que otros patógenos están evolucionando directamente desde las megagranjas (Wallace et al., 2019). Con la globalización y la alta conectividad social, los patógenos se transfieren rápidamente desde lugares remotos a los centros urbanos, y en estos últimos se expanden aceleradamente las enfermedades que ellos producen, como es el caso de la Covid-I9.

Con esos antecedentes, la producción de alimentos debe planificarse teniendo en cuenta el equilibrio entre las interacciones de los ciclos biogeoquímicos y energéticos de las explotaciones agropecuarias dentro de un marco geográfico biorregional. Para tal fin, se debe avanzar hacia prácticas agroecológicas que superen los impactos del monocultivo agroindustrial (Altieri et al., 2015), pero ellas deben ser analizadas desde una perspectiva multicriterio para evaluar su viabilidad y sus consecuencias (Padró et al., 2020). 


\section{El desarrollo territorial pos Covid-19}

En el escenario pos Covid-19 la economía estará en el centro de la agenda política global. Desde esta Tribuna se llama a evitar las consecuencias ya experimentadas en las salidas de anteriores crisis económicas, en las cuales se incrementaron las emisiones de GEI y los efectos negativos sobre el ambiente, al implementar medidas principalmente pensadas en la recuperación económica (Peters et al., 2012). Igualmente, es necesario tener presente que el actual acento en la denominada "economía circular" puede generar un efecto "rebote" en el que las actividades económicas que tienen menores impactos ambientales negativos por unidad de producción, sean la causa de un aumento en los niveles productivos y, por ende, una reducción en los beneficios ambientales (Zink \& Geyer, 2017). Por ello, asumimos el llamado de la Declaración Vasca (2016) a la transformación sociocultural, socioeconómica y tecnológica, sobre la base de un cambio en los medios de producción y consumo de bienes y servicios, reconociendo la existencia de límites biofísicos, manteniendo la funcionalidad de los ecosistemas y optimizando el aprovechamiento de los recursos naturales disponibles. La actual crisis ambiental, y en general la crisis sistémica global acelerada por la pandemia de la covid-19, supone una oportunidad para avanzar hacia una redefinición del territorio en clave de protección de la salud humana, y la de los ecosistemas para aumentar su resiliencia frente a los riesgos antropogénicos como los previamente referenciados.

En las secciones anteriores se ha ilustrado cómo la alteración del paisaje desde las acciones asociadas a los modelos urbano-rurales preponderantes ha venido provocando riesgos de alto costo social y ambiental. El caso de la covid-19 ha mostrado que su letalidad ha sido mayor en poblaciones con patologías previas relacionadas con los modelos analizados. Si bien las relaciones de causa-efecto entre los cambios ambientales y las afecciones sanitarias no son tan fáciles de establecer, la Evaluación de los Ecosistemas del Milenio identificó la relación de los servicios ecosistémicos con el bienestar y la salud humana. Existe evidencia científica sobre la capacidad de los ecosistemas para adaptarse a los cambios, y dada la diversidad que albergan, su contribución a regular las enfermedades. A mayor biodiversidad, más dificultad de propagación de un patógeno (UNEP, 2016). También, hay evidencias que señalan que la relación costo/beneficios en la reducción de contaminación atmosférica es positiva no solo para la salud humana, sino también para el aumento de la producción agrícola y la reducción de la pérdida de biodiversidad, relación que también es extensible al acceso al agua potable y al saneamiento (Elkins et al., 2019). Dicha evidencia resulta fundamental para avanzar hacia una redefinición de las relaciones campo-ciudad.

En ese contexto, se considera necesario que la planificación territorial avance hacia el mantenimiento y restauración de su estructura ecológica de soporte, como expresión de los procesos que sustentan la vida humana, la biodiversidad y el suministro de servicios ambientales a largo plazo (van der Hammen \& Andrade, 2003). Para ello, la Estrategia de Infraestructuras Verdes en Europa (Comisión Europea, 2013) y países como Espańa (Gobierno de Espańa, 2020) se presenta como un marco adecuado para la planificación, ya que dichas infraestructuras se conciben como una 
red de zonas naturales y seminaturales destinadas a integrar tanto la biodiversidad como su multifuncionalidad de servicios ecosistémicos. Así pues, se reivindica la necesidad de avanzar hacia una redefinición territorial en clave biorregional, donde se delimiten ámbitos espaciales con un significado ecológico y cultural, caracterizados por rasgos semejantes en lo biofísico y socioeconómico. Aquí desempeña un rol central la apropiación del sistema patrimonial natural, constituido por el conjunto de bienes culturales o inmateriales, naturales o antrópicos que ostentan un valor social, buscando la maximización de la calidad del flujo de servicios ecosistémicos en la nueva configuración territorial (Sánchez-Fuentes \& Mascort-Albea, 2021). El reto es que la biorregión tienda a la resiliencia como socioecosistema, buscando el equilibrio territorial desde una organización policéntrica y modular; es decir, desde sistemas a partir de módulos o estructuras conectadas, pero no superpuestas. Cada uno de estos módulos se debe concebir como una entidad flexible y capaz de adaptarse a las condiciones externas, sin que las perturbaciones arrastren al conjunto del ecosistema (Walker \& Salt, 2006). Las transformaciones a pequeña escala son más manejables, se favorecen de la resiliencia de las múltiples escalas jerárquicas y pueden beneficiarse de la experiencia y conocimiento adquiridos en crisis para avanzar hacia transiciones socioecológicas (Folke et al., 2010).

Partir de una concepción del territorio como un sistema socioecológico permite integrar tanto los elementos relacionados con el desarrollo territorial, la producción y consumo, como los impactos que las actividades humanas tienen en la explotación de los recursos naturales y la alteración de los ecosistemas y la biodiversidad. El reto actual estriba en que los enfoques para el análisis de los sistemas socioecológicos presentan diferencias significativas en su conceptualización e interrelación (Binder et al., 2013), lo cual dificulta su aplicación desde un punto de vista transdisciplinario y práctico. En ese contexto, toma fuerza el enfoque de metabolismo social, como una herramienta robusta utilizada para comprender la compleja realidad de los sistemas socioecológicos y su coevolución histórica (González de Molina \& Toledo, 2014). Este marco supone la coexistencia de diferentes escalas y dimensiones de análisis que permiten caracterizar la reproducción de los seres humanos mediante un flujo continuo de energía y materiales tomados y desechados al ambiente (Giampietro et al., 2009). Desde dicha herramienta se pueden realizar análisis integrados de las trayectorias metabólicas de posibles cambios socioecológicos, incluyendo escenarios de desarrollo territorial con restricciones biofísicas y el menor consumo energético. Muestra de su utilidad es el reciente estudio de Padró et al. (2020), en el cual se cuantificaron los flujos metabólicos de los usos del suelo de infraestructura verde, con el objeto de soportar decisiones en la conciliación del desarrollo urbano y actuaciones en los espacios abiertos circundantes del área metropolitana de Barcelona, considerando su Plan Director Urbanístico.

Sobre la base de todo lo expuesto con anterioridad, se puede concluir que la capacidad de articulación y fomento de la identidad territorial biorregional ayudará a mejorar la funcionalidad de sus componentes como infraestructuras verdes, como conectores para la biodiversidad, y como elementos de adaptación al cambio climático (Gallardo Ramírez, 2019). La clave está en que el desarrollo de políticas de ordenación territorial, como la de las infraestructuras verdes, esté acompañada 
de políticas económicas que permitan una planificación integrada y coherente con la construcción de resiliencia socioecológica. Ello permitiría avanzar hacia una redefinición de las relaciones de los modelos urbanos y rurales basada en las potencialidades y limitaciones biofísicas existentes, con una transición a espacios híbridos dinámicos, adaptativos y equipados para una comunidad arraigada al lugar y disfrutando del derecho colectivo a un ambiente sano. En ese sentido, resulta imprescindible fortalecer la toma de conciencia masiva sobre la problemática enunciada, cuya raíz fundamental quedó reflejada hace décadas en los versos del poeta Nicanor Parra (1982): "El error consistió en creer que la tierra era nuestra cuando la verdad de las cosas es que nosotros somos de la tierra".

\section{Referencias bibliográficas}

Altieri, M. A., Nicholls, C. I., Henao, A. \& Lana, M. A. (2015). Agroecology and the design of climate change-resilient farming systems. Agronomy for sustainable development, 35(3), 869-890. https://doi.org/10.1007/s13593-015-0285-2

Binder, C. R., Hinkel, J., Bots, P. W. \& Pahl-Wostl, C. (2013). Comparison of frameworks for analyzing social-ecological systems. Ecology and Society, 18(4), 26. http://dx.doi. org/10.5751/ES-05551-180426

Bruinsma, J. (Ed.). (2003). World agriculture: towards 2015/2030: an FAO perspective. Earthscan. http://www.fao.org/3/y4252e/y4252e00.htm

Centre for Research on the Epidemiology of Disasters \& United Nations Office for Disaster Risk Reduction (CRED \& UNDRR). (2020). Human cost of disasters: an overview of the last 20 years (2000-2019). CRED / UNDRR. https://bit.ly/39EkvMK

Comisión Europea. (2013). Comunicación de la Comisión al Parlamento Europeo, al Consejo, al Comité económico y social europeo y al Comité de las regiones. Infraestructura verde: mejora del capital natural de Europa. Bruselas, 6.5.2013, сом (2013) 249 final. https://bit.ly/3bBJClP

Declaración Vasca. (2016). Nueva hoja de ruta para ciudades y pueblos europeos, creando municipios productivos, sostenibles y resilientes para una Europa habitable e inclusiva. 8th European Conference on Sustainable Cities and Towns, Bilbao, España, 27/04/2016 a 29/04/2016. https://sustainablecities.eu/fileadmin/repository/Basque_Declaration/ Basque-Declaration-SPANISH-WWW.pdf

Elkins, P., Gupta, J. \& Boileau, P. (Eds.). (2019). Global Environment Outlook: GEO-6: Healthy Planet, Healthy People. Cambridge University Press. http://hdl.handle. net/20.500.11822/27539

Foley, J. A., DeFries, R., Asner, G. P., Barford, C., Bonan, G., Carpenter, S. R., Chapin, F. S., Coe, M. T., Daily, G. C., Gibbs, H. K., Helkowski, J. H., Holloway, T., Howard, E. A., Kucharik, C. J., Monfreda, C., Patz, J. A., Prentice, I. C., Ramankutty, N. \& Snyder, P. K. (2005). Global consequences of land use. Science, 309(5734), 570-574. http://doi.org/10.1126/science.1111772 
Folke, C., Carpenter, S. R., Walker, B., Scheffer, M., Chapin, T. \& Rockström, J. (2010). Resilience thinking: Integrating resilience, adaptability and transformability. Ecology and Society, 15(4). http://www.ecologyandsociety.org/vol15/iss4/art20/

Food and Agricultural Organization (FAO). (2015). Climate change and food systems: Global assessments and implications for food security and trade. Editado por A. Elbehri. FAO. http://www.fao.org/3/a-i4332e.pdf

Gallardo Ramírez, C. (2019). Método de evaluación para la regeneración integrada del espacio turístico litoral: la Costa del Sol Occidental [Tesis Doctoral, Universidad de Sevilla]. https://hdl.handle.net/11441/81513

Giampietro, M., Mayumi, K. \& Ramos-Martin, J. (2009). Multi-scale integrated analysis of societal and ecosystem metabolism (Musiasem): Theoretical concepts and basic rationale. Energy, 34(3), 313-322. https://doi.org/10.1016/j.energy.2008.07.020

Gobierno de España. (2020). Estrategia Nacional de Infraestructura Verde y de la Conectividad y Restauración Ecológicas. Vicepresidencia cuarta del Gobierno, Ministerio para la transición ecológica y el reto demográfico. https://bit.ly/2N9mSzz

González de Molina, M. \& Toledo, V. M. (2014). The social metabolism: a socio-ecological theory of historical change. Environmental History Vol. 3, Springer.

Gravlee, C. C. (2020). Systemic racism, chronic health inequities, and covid-19: A syndemic in the making? American Journal of Human Biology 32(5), e23482. https://doi. org/10.1002/ajhb.23482

Kapecki, T. (2020). Elements of sustainable development in the context of the environmental and financial crisis and the covid-19 pandemic. Sustainability, 12(15), 6188. https:// doi.org/10.3390/su12156188

Lelieveld, J., Pozzer, A., Pöschl, U., Fnais, M., Haines, A. \& Münzel, T. (2020). Loss of life expectancy from air pollution compared to other risk factors: a worldwide perspective. Cardiovascular Research, 116(11), 1910-1917. https://doi.org/10.1093/cvr/cvaa025

Megahed, N. A. \& Ghoneim, E. M. (2020). Antivirus-built environment: Lessons learned from Covid-19 pandemic. Sustainable Cities and Society, 61, 102350. https://doi. org/10.1016/j.scs.2020.102350

Millennium Ecosystem Assessment. (2005). Ecosystems and Human Well-being: Synthesis. Island Press. http://www.millenniumassessment.org/documents/document.356.aspx.pdf

Moore, M., Gould, P. \& Keary, B. S. (2003). Global urbanization and impact on health. International journal of hygiene and environmental health, 206(4-5), 269-278. https:// doi.org/10.1078/1438-4639-00223

Padró, R., La Rota-Aguilera, M. J., Giocoli, A., Cirera, J., Coll, F., Pons, M., Pino, S. P., Serrano, T., Villalba, G. \& Marull, J. (2020). Assessing the sustainability of contrasting land use scenarios through the Socioecological Integrated Analysis (SIA) of the metropolitan green infrastructure in Barcelona. Landscape and Urban Planning, 203, 103905. https://dx.doi.org/10.1016\%2Fj.landurbplan.2020.103905

Parra, N. (1982). Ecopoemas. Gráfica Marginal.

Peters, G. P., Marland, G., Le Quéré, C., Boden, T., Canadell, J. G. \& Raupach, M. R. (2012). Rapid growth in CO2 emissions after the 2008-2009 global financial crisis. Nature Climate Change, 2(1), 2-4. https://doi.org/10.1038/nclimate1332 
Prats, F., Cuchí, A. \& Oscariz, J. (2017). Ante el antropoceno. Reflexiones sobre la cuestión biorregional en el País Vasco. Dpto. de Medio Ambiente, Planificación Territorial y Vivienda. Gobierno Vasco. https://bit.ly/3oKnklr

Sánchez-Fuentes, D. \& Mascort-Albea, E. J. (2021). Metropolitan metabolism: the ecological footprint. En A. Contin, P. Giordano \& M. Nacke (Eds.), Training for education, learning and leadership towards a new metropolitan discipline. Inaugural book (pp. 4146). Centro de Implementación de Políticas Públicas para la Equidad y el Crecimiento (CIPPEC). https://www.cippec.org/wp-content/uploads/2021/02/TELLme-Inaugu ralBook-1.pdf

Spaiser, V., Ranganathan, S., Swain, R. B. \& Sumpter, D. J. (2017). The sustainable development oxymoron: quantifying and modelling the incompatibility of sustainable development goals. International Journal of Sustainable Development \& World Ecology, 24(6), 457470. https://doi.org/10.1080/13504509.2016.1235624

United Nations Environmental Programme (UNEP). (2016). UNEP Frontiers 2016 Report: Emerging Issues of Environmental Concern. United Nations Environmental Programme, Nairobi. https://wedocs.unep.org/handle/20.500.11822/7664

United Nations, Department of Economic and Social Affairs (Un-DesA). (2019). World Population Prospects 2019: Highlights. un-DESA, Population Division. sT/ESA/sER.A/423. https://population.un.org/wpp/Publications/Files/WPP2019_Highlights.pdf

van der Hammen, T. \& Andrade, G. (2003). Estructura ecológica principal de Colombia: Primera aproximación. Ministerio de Ambiente, Vivienda y Desarrollo Territorial, Instituto de Hidrología, Meteorología y Estudios Ambientales, Bogotá, Colombia. http:// observatorio.epacartagena.gov.co/ftp-uploads/pub-Estructuraecologicaprincipal.pdf

Vitousek, P. M., Mooney, H. A., Lubchenco, J. \& Melillo, J. M. (1997). Human domination of Earth's ecosystems. Science, 277(5325), 494-499. https://doi.org/10.1126/ science.277.5325.494

Walker, B. \& Salt, D. (2006). Resilience thinking: Sustaining ecosystems and people in a changing world. Island Press.

Wallace, R. G., Alders, R., Kock, R., Jonas, T., Wallace, R. \& Hogerwerf, L. (2019). Health before medicine: Community resilience in food landscapes. One Planet-Eco-One Health: Looking After Humans, Animals and the Environment, Sydney University Press.

Wallace, R., Liebman, A., Chaves, L. F. \& Wallace, R. (2020). covid-19 and Circuits of Capital. Monthly Review, 72(1), 1-13. https://monthlyreview.org/2020/05/01/covid19-and-circuits-of-capital/

Yach, D., Stuckler, D. \& Brownell, K. D. (2006). Epidemiologic and economic consequences of the global epidemics of obesity and diabetes. Nature medicine, 12(1), 62-66. https:// doi.org/10.1038/nm0106-62

Zink, T. \& Geyer, R. (2017). Circular economy rebound. Journal of Industrial Ecology, 21(3), 593-602. https://doi.org/10.1111/jiec. 12545 